\title{
Evidence supporting a role for circulating macrophages in the regression of vascular remodeling following sub-chronic exposure to hemoglobin plus hypoxia
}

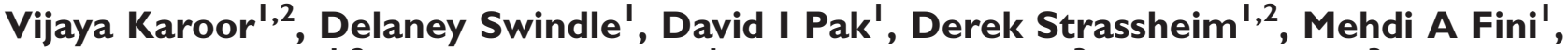 \\ Edward Dempsey ${ }^{1,2}$, Kurt R Stenmark' (D, Kathryn Hassell ${ }^{3}$, Rachelle Nuss ${ }^{3}$, \\ Paul W. Buehler ${ }^{4,5}$ (D) and David C. Irwin' (D) \\ 'Cardiovascular and Pulmonary Research Laboratory, Department of Medicine, University of Colorado Denver, Anschutz Medical Campus, Aurora, CO, USA; \\ ${ }^{2}$ Pulmonary Sciences and Critical Care Medicine, Department of Medicine, University of Colorado Denver, Anschutz Medical Campus, Aurora, CO, USA; ${ }^{3}$ Division \\ of Hematology Colorado Sickle Cell Treatment and Research Center, School of Medicine, Anschutz Medical Campus, University of Colorado-Denver School of \\ Medicine, Aurora, CO, USA; ${ }^{4}$ Department of Pathology, University of Maryland, Baltimore, MD, USA; ${ }^{5}$ The Center for Blood Oxygen Transport, Department of \\ Pediatrics, School of Medicine, Baltimore, MD, USA
}

\begin{abstract}
Macrophages are a heterogeneous population with both pro- and anti-inflammatory functions play an essential role in maintaining tissue homeostasis, promoting inflammation under pathological conditions, and tissue repair after injury. In pulmonary hypertension, the MI phenotype is more pro-inflammatory compared to the $\mathrm{M} 2$ phenotype, which is involved in tissue repair. The role of macrophages in the initiation and progression of pulmonary hypertension is well studied. However, their role in the regression of established pulmonary hypertension is not well known. Rats chronically exposed to hemoglobin (Hb) plus hypoxia ( $\mathrm{HX})$ share similarities to humans with pulmonary hypertension associated with hemolytic disease, including the presence of a unique macrophage phenotype surrounding distal vessels that are associated with vascular remodeling. These lung macrophages are characterized by high iron content, HO-I, ET-I, and IL-6, and are recruited from the circulation. Depletion of macrophages in this model prevents the development of pulmonary hypertension and vascular remodeling. In this study, we specifically investigate the regression of pulmonary hypertension over a four-week duration after rats were removed from $\mathrm{Hb}+\mathrm{HX}$ exposure with and without gadolinium chloride administration. Withdrawal of $\mathrm{Hb}+\mathrm{HX}$ reversed systolic pressures and right ventricular function after $\mathrm{Hb}+\mathrm{Hx}$ exposure in four weeks. Our data show that depleting circulating monocytes/macrophages during reversal prevents complete recovery of right ventricular systolic pressure and vascular remodeling in this rat model of pulmonary hypertension at four weeks post exposure. The data presented offer a novel insight into the role of macrophages in the processes of pulmonary hypertension regression in a rodent model of $\mathrm{Hb}+\mathrm{Hx}$-driven disease.
\end{abstract}

\section{Keywords}

lung, heart, sickle cell disease, pulmonary vascular disease, hemoglobinopathies

Date received: 29 July 202I; accepted: 12 October 2021

Pulmonary Circulation 202 I; I I (4) I-I I

DOI: I0.II77/204589402 I I056806

\section{Introduction}

Pulmonary hypertension $(\mathrm{PH})$ is a multi-factorial disease leading to progressive right heart failure and death. ${ }^{1}$ The primary pathophysiological parameter that defines $\mathrm{PH}$ is a mean pulmonary arterial (PA) pressure exceeding $25 \mathrm{~mm} \mathrm{Hg}^{2}$ and PH occurs in a variety of clinical situations categorized into six groups by the World Health Organization (WHO). ${ }^{1,2}$ The type of $\mathrm{PH}$ that manifests in

\footnotetext{
*These authors contributed equally to this manuscript.

Corresponding author:

David C. Irwin, University of Colorado Denver, Anschutz Medical Campus, Research Building 2, BI33, Room 8I2I, Aurora, CO 80045, USA.

Email: david.irwin@cuanschutz.edu
} 
patients with hemolytic anemias such as sickle cell disease (SCD) is classified to group 5, where $\mathrm{PH}$ is secondary to either pre- $(52.6 \%$ of patients) or post-capillary driven disease $(47.4 \%$ of patients) and is the primary cause of death. In SCD patients, pre- or post-capillary-driven $\mathrm{PH}$ processes are causally linked to the pulmonary vasculopathy caused by free hemoglobin $(\mathrm{Hb})$ and its degradation products, heme, and iron, resulting in nitric oxide depletion hypoxia and macrophage reprogramming. ${ }^{3,4}$ We have developed an animal model that combines chronic $\mathrm{Hb}$ infusion and hypoxia (HX) to mimic intravascular hemolysis and impaired tissue oxygenation to approximate $\mathrm{PH}$ in the SCD population. ${ }^{5-7}$

An increase in interstitial and perivascular macrophages is observed in rodent and human $\mathrm{PH}$ and is implicated in the pathogenesis and vascular remodeling. Macrophages also play a significant role in removing $\mathrm{Hb}$ and heme through haptoglobin and hemopexin trafficking and senescent red blood cell removal. However, excess macrophage iron can increase iron extrusion into the physiological environment. ${ }^{8}$ In our PH model, we have observed a macrophage phenotype characterized by intracellular accumulation of iron, robust expression of heme oxygenase 1 (HO-1), and mediators of hypoxia (HX)-induced PH such as endothelin-1 (ET-1) and interleukin 6 (IL-6). Furthermore, by depleting circulating macrophages with gadolinium trichloride $\left(\mathrm{GdCl}_{3}\right)$, we observed a significant attenuation of the $\mathrm{PH}$ response, suggesting a critical role for macrophages in the development and progression of $\mathrm{PH}$ during exposures to $\mathrm{Hb}$ and $\mathrm{HX}^{7}$

Macrophages are essential in the resolution of inflammation and tissue repair after injury. However, there is a dearth of studies addressing the role of macrophages in regression of established $\mathrm{PH}$. The present study is designed to determine whether iron-laden macrophages persist after cessation of $\mathrm{Hb}$ and concomitant $\mathrm{Hx}$ exposure. Further, we sought to determine if macrophages have a role in reversing medial hypertrophy of pulmonary vasculature. While this study is an observational examination of $\mathrm{PH}$ regression in rats after $\mathrm{Hb}$ plus $\mathrm{HX}$ exposure, we also ask a critical prospective question. We hypothesized that a marked reduction in adventitial macrophages rich in iron, HO-1, IL-6, and ET-1 would occur within a month after cessation of $\mathrm{Hb}$ plus $\mathrm{HX}$, and the elimination of circulating macrophages with Gadolinium Chloride $\left(\mathrm{GdCl}_{3}\right)$ would slow the recovery process. This hypothesis is based on the insufficiently explored role of macrophages in the reversal of pulmonary vascular remodeling. Data described herein define the processes in a relevant animal model of group $5 \mathrm{PH}$.

\section{Materials and methods}

\section{Animals}

Male Sprague-Dawley rats were obtained from a commercial vendor (Jackson Laboratories, Bar Harbor, ME, USA).
All experimental protocols were reviewed and approved by the Institutional Animal Care and Use Committee at our University. All animals survived the surgical procedure and indwelling catheterization into the jugular vein. None of the rats exhibited any signs or symptoms indicative of systemic infection. After surgery, wounds healed within 14 days.

Rats $(n=28)$ were randomly assigned to one of four groups: (1) normoxic control (NX; $\mathrm{n}=6$ ); (2) hypoxic control (HX; $\mathrm{n}=6)$; (3) hemoglobin plus hypoxia $(\mathrm{Hb}+\mathrm{HX}$; $\mathrm{n}=8$ ); (4) hemoglobin plus hypoxia treated with gadolinium chloride $\left(\mathrm{Hb}+\mathrm{HX}+\mathrm{GdCl}_{3} ; \mathrm{n}=8\right)$.

\section{iPrecio infusion pump placement}

Programmable infusion pumps (iPRECIO, Tokyo, Japan) were placed subcutaneously as previously described. ${ }^{5}$ iPRECIO pumps were programmed to deliver $\mathrm{Hb}(35 \mathrm{mg} /$ day or $250 \mathrm{ug} / \mathrm{uL}$ ) or saline at an infusion rate of $6 \mathrm{~mL} / \mathrm{h}$ for 35 days. Pumps were refilled with fresh aliquots of $\mathrm{Hb}$ or saline every three to four days. At a dose of $35 \mathrm{mg} /$ day, we show that total plasma heme concentrations were in the range of values similar to a mild to moderate chronic hemolytic state observed in $\mathrm{SCD} .^{5,6}$

\section{Endotoxin and catalase-free human adult hemoglobin $\left(\mathrm{HbA} ;\left(\mathrm{Fe}^{2+}\right)\right)$}

Purified human endotoxin-free $\mathrm{Hb}$ (LPS $<0.5$ endotoxin units, EU) was prepared from outdated blood as previously described, with an additional step to remove catalase by chromatography. ${ }^{9}$ Approximately $1 \mathrm{~g}$ of $\mathrm{Hb}$ protein was loaded onto a GE Healthcare glass column (HR 16/50:1 $\left.1 / 2^{\prime \prime} \times 26^{\prime \prime}\right)$ packed with SuperoseÔ 6 (Code No. 17-0489-01, Amersham Biosciences, Upsala, Sweden) and run at $4{ }^{\circ} \mathrm{C}$ and a flow rate of $2 \mathrm{~mL} / \mathrm{h}$. $\mathrm{Hb}$ protein was collected and was followed by buffer switching to $0.1 \mathrm{NaCl}$ to remove catalase from the column. Several runs were pooled and concentrated to $200 \mathrm{mg} / \mathrm{mL}$ using Centricon Plus-70 with $30 \mathrm{kDa}$ cutoff membrane filters (Millipore, Billerica, MA, USA). The starting composition of $\mathrm{Hb}$ was $96.5 \pm 1.3 \%$ $\mathrm{Fe}^{2+}, 3.50 \pm 0.23 \% \mathrm{Fe}^{3+}$, and no measurable hemichrome. Endotoxin concentration was $<0.5 \mathrm{EU} / \mathrm{mL}$ as determined by the limulus ambocyte lysate assay and failed to activate macrophages in culture.

\section{Hypoxia exposure}

Rats were continuously exposed to simulated high altitude $(5500 \mathrm{~m}, 18,000 \mathrm{ft})$ in a rodent hypobaric chamber facility as previously described. ${ }^{5-7}$ Animals were exposed to this atmosphere continuously for 35 days. It has been previously demonstrated that chronic hypoxia can increase pulmonary arterial pressure, right ventricular hypertrophy, and pulmonary vascular remodeling. 


\section{Echocardiography}

To assess approximate time frames of the progression and resolution of $\mathrm{PH}$ echocardiographic analysis was performed at two-week intervals using a high-resolution in vivo microimaging Vevo770 system (Visual-Sonics). Care was taken to follow the guidelines for measuring cardiac physiology in rats established by the American Physiological Society. ${ }^{10}$ For each analysis, rats were anesthetized via an isoflurane $/ \mathrm{O}_{2}$ mixture with induction at $4 \%$ and maintenance between $1.5 \%$ and $3 \%$, and body temperature was maintained at $37^{\circ} \mathrm{C}$ via a heated surgical pad. As described by Cavasin et al., ${ }^{11}$ pulse-wave Doppler imaging of the pulmonary outflow was recorded in the parasternal short-axis view at the level of the aortic valve. Animals were then monitored during the recovery from anesthesia; upon full recovery, they were returned to their respective housing

\section{Gadolinium chloride}

Gadolinium chloride $\left(\mathrm{GdCl}_{3}\right)$ (Cat \# G7532; Sigma) was dissolved in sterile $0.9 \% \mathrm{NaCl}$ and delivered intravenously in a $1 \mathrm{~mL}$ bolus. Rats were treated with gadolinium chloride every three days at a dose of $10 \mathrm{mg} / \mathrm{kg}$ for four weeks. We previously demonstrated by qRTPCR analysis that this dose effectively depletes macrophages by depleting $\mathrm{CD}_{163}{ }^{+}$and $\mathrm{CD} 68^{+}$cells in the liver. ${ }^{7}$

\section{Endpoint blood pressure measurements}

Pulmonary blood pressures were measured with a $1.9 \mathrm{~F}$ pressure-volume catheter placed in the main PA in an open-chest procedure. Correct placement of the catheter was confirmed by observing the normal rise in diastolic pressure as the catheter was moved into the PA. Systemic blood pressure was monitored with a catheter inserted in the femoral artery. Cardiac performance was assessed using an ADV500 pressure-volume system (Transonic/Scisense), and data acquired and analyzed with Labscribe2 (iWorx). For all measurements, animals were anesthetized with $5 \%$ and maintained at $2 \%$ isoflurane, and body temperature was maintained at $37^{\circ} \mathrm{C}$.

\section{Blood and organ collection}

Serial blood draws were conducted at baseline (pump placement) and every three days post initiation of $\mathrm{Hb}$ and hypoxia exposure. Blood was placed in an EDTA$\mathrm{K}+$ vacutainer and a crit tube to analyze plasma and hematocrit, respectively.

Animals were exsanguinated via a carotid artery catheter; blood was placed in a vacutainer with EDTA and centrifuged. The plasma was collected, snap-frozen in liquid nitrogen, and stored at $-80^{\circ} \mathrm{C}$ until analysis; $300 \mu \mathrm{L}$ of blood was also placed in a crit-tube and spun to measure hematocrits. The femoral artery was severed, and the lungs, kidneys, spleen, and liver were perfused with PBS (120mL) via the right ventricle to remove blood. The kidneys, spleen, and liver were cut in two sections: one piece was placed in $10 \%$ formalin and the other in AllProtect (Qiagen, Valencia, CA, USA). The right lung lobes were tied off at the right main bronchus, removed, and a piece was placed in AllProtect, and another portion was snap-frozen. The left lung was fixed with $10 \%$ formalin $(\sim 3 \mathrm{~mL})$ by airway inflation under constant pressure at $25 \mathrm{~cm} \mathrm{H}_{2} \mathrm{O}$ pressure, and the entire lung-heart bloc was removed. The hearts were removed, and the right and left ventricles plus septum were isolated. The right ventricle (RV) and left ventricle with the septum $(\mathrm{LV}+\mathrm{S})$ were weighed for assessment of the Fulton Index (right ventricular hypertrophy; RV/ $\mathrm{LV}+\mathrm{S}$ ). After $24 \mathrm{~h}$, the formalin-fixed organs were removed from $10 \%$ formalin and placed in $70 \%$ ethanol, and prepared following standard methods for morphometric and immunohistochemical analyses.

\section{Materials}

The following antibodies were used: $\mathrm{b}$ actin (cat \# ab8226), 1:1000 (Abcam); smooth muscle actin (cat \#ab5694 and ab7817), 1:1000 (Abcam); heme oxygenase (HO1; cat \# ab13248), 1:250 (Abcam); interleukin 6 (IL6; cat \# ab9324), 1:1000 (Abcam); endothelin 1 (ET1; cat \# ab117757), 1:500 (Abcam); CD68 (cat \# ab955), 1:500 (Abcam); intracellular adhesion molecule 1 (ICAM; cat \# ab2213), 1:250 (Abcam). Lung sections were incubated with a fluorescent secondary antibody either AlexaFluor 488; 1:1000 or AlexaFluor 555; 1:1000 (Invitrogen; Carslbad, CA, USA) or a biotinylated secondary horse anti mouse or horse anti-rabbit, 1:1000 (Vector Labs, Burlingame, CA, USA).

\section{Western blot analysis}

Lungs were homogenized using a TissueMiser (ThermoFisher, Waltham, MA, USA) in RIPA buffer containing protease inhibitors; protein concentrations were determined using a Pierce BCA Protein Assay. Briefly, Western blot analysis was performed using $30 \mathrm{mg}$ of sample protein run under denaturing and reducing conditions on Biorad Criterion Tris- $\mathrm{HCl} 12.5 \%$ gels with a Biorad SDS-Page blot system. All proteins were normalized to b-actin. Statistical analysis was determined from the fold difference from $\mathrm{Nx}$ (normoxic control) on the same gel. Gels were imaged on an Alpha Innotech gel documentation system (Protein Simple, Santa Clara, CA, USA), and densitometric analyses were performed using ImageJ software (version $1.44 \mathrm{o}$, National Institutes of Health, USA).

\section{Immunohistochemistry and morphology}

Formalin-fixed, paraffin-embedded lung, kidney, and spleen tissue was sectioned by our university. Five-micrometer sections were stained with hematoxylin and eosin by standard procedures to assess the accumulation of 
perivascular cells as well as vessel wall thickness. Lung sections were also stained for smooth muscle actin utilizing a biotinylated horse anti-mouse immunoglobulin followed by a 3,3'-diaminobenzidine (DAB) for development to quantify small arterial muscularization.

The entire tissue field on each slide was scanned utilizing a Leica AperioCS2 microscope. Analyses of the scanned images were performed using the Aperio eSlide manager software (Leica, Buffalo Grove, IL, USA). Positively stained smooth muscle vessels were counted and described as either fully or partially muscularized. Any area that fell outside of the lung boundary was subtracted from the total area, and the number of either fully or partially muscularized vessels was normalized to area. Distal pulmonary vessels (outside diameter of $10-50 \mathrm{~mm}$ ) were assessed for degree of circumferential a-smooth muscle actin-positive staining, indicative of muscularization. Proximal vessels (outside diameter of 50-250 $\mathrm{mm}$ ) were analyzed for medial wall thickness at four points around the vessel circumference and for lumen diameter along two axes. Wall thickness is expressed as the ratio of medial wall thickness to lumen radius.

Histological analysis of nonheme iron deposition in lung tissue was detected using Perl's staining with DAB counterstain as previously described. ${ }^{5-7}$ For quantification, a total of 20 images at $20 \times$ magnification were obtained from sections of all animals. Within each of the 20 images, the total number of Perl's positive cells was counted and divided by 20 .

\section{Statistical analysis}

For all groups, the mean SEM is reported. Statistical comparisons for data measurements were completed with either one-way analysis of variance ${ }^{12}$ for $\mathrm{NX}, \mathrm{HX}, \mathrm{Hb}+\mathrm{HX}$, and $\mathrm{Hb}+\mathrm{HX}+\mathrm{GdCl}_{3}$ treatment (endpoint analysis) or twoway ANOVA for analysis between time (echocardiography analysis). Echocardiography data were also analyzed by a univariate repeated measures test using JMP (SAS Cary, NC, USA). All Post hoc analyses were completed with unpaired, two-sided Student $t$-test with a Bonferroni correction unless otherwise noted. Statistical analyses were performed using GraphPad Prism (La Jolla, CA, USA) with statistical significance set at $p \leq 0.05$.

\section{Results}

\section{Macrophage depletion slows the resolution of pulmonary vascular remodeling in a hemoglobin plus hypoxia rat model of pulmonary hypertension}

Hypoxia-induced vascular remodeling reverses upon reoxygenation. We demonstrate that rats exposed to HX with continuous low-level $\mathrm{Hb}$ infusion for five weeks develop a more severe $\mathrm{PH}$ than with $\mathrm{Hx}$ alone..$^{5-7}$ In this study, we wanted to assess whether our PH model would be reversible after the withdrawal of $\mathrm{Hb}$ and $\mathrm{HX}$ and if macrophages have a role in this process. To this end, circulating monocytes/macrophages were depleted with $\mathrm{GdCl}_{3}$ during a fourweek recovery period after cessation of both $\mathrm{Hb}$ and $\mathrm{HX}$ (Fig. 1a). Groups consisted of normoxic control (NX), hypoxic control (HX5wk), (Hb + HX5wk), and HX 5wk followed by four weeks normoxia (HX5wk; NX+4wk), $\mathrm{Hb}+\mathrm{HX} 5 \mathrm{wk}$ followed by four weeks no $\mathrm{Hb}$ infusion in normoxia $(\mathrm{Hb}+\mathrm{HX} 5 \mathrm{wk} ; \quad-\mathrm{Hb}+\mathrm{HX} 4 \mathrm{wk}) \pm \mathrm{GdCl}_{3}$ (Fig. 1b). Serial echocardiography and endpoint hemodynamics, Fulton index $(\mathrm{RV} / \mathrm{LV}+\mathrm{S})$, and lung morphology/ histology were used to assess the impact of macrophage depletion on PH resolution. The purpose of echocardiography was to understand approximate time frames of the progression and resolution of $\mathrm{PH}$, and not as a primary indicator to identify exact differences among groups more suitable for endpoint analysis.

Echocardiography analysis revealed that $\mathrm{Hb}+\mathrm{HX}$ or HX exposed rats demonstrated increased pulmonary artery acceleration time (PAAT) and RV wall thickness compared to NX cohorts at five weeks. Combining all $\mathrm{Hb}+\mathrm{HX}$ rats at five weeks before $\mathrm{GdCl}_{3}$ treatment revealed lower PAAT in $\mathrm{Hb}$ plus $\mathrm{HX}$ rats than rats only exposed to $\mathrm{Hx}$, reflecting higher PA pressures (Fig. 1b, inset) and more progressive PH (Fig. 1b-d). This main effect of $\mathrm{Hb}+\mathrm{HX}$ is congruent with our previous studies demonstrating higher pulmonary arterial pressures in $\mathrm{Hb}$ plus HX rats vs. HX alone. ${ }^{5,6}$ Analysis of serial echocardiography data shows a significant improvement of PAAT and RV wall thickness during the four-week recovery period (Fig. 1b-d). However, rats maintained on continuous $\mathrm{Hb}$ infusion plus HX had lower PAAT and greater RV wall thickness at two- and four-week time points of recovery (Fig. 1b and c) than HX alone rats (Fig. 1b-d).

Invasive hemodynamic measurements obtained after four weeks of recovery show the elimination of circulating macrophages with $\mathrm{GdCl}_{3}$ prevented the complete resolution of mean pulmonary artery pressure (PAP) and pulmonary vascular remodeling as compared between the $\mathrm{Hb}+\mathrm{HX}$ and $\mathrm{Hb}+\mathrm{HX}+\mathrm{GdCl}_{3}$ groups (Fig. 1e-h). These data suggest that a subset of circulating monocytes/macrophages recruited to the lung may be required for the complete regression of $\mathrm{Hb}+\mathrm{HX}$-induced vascular remodeling. While the Fulton index calculation $(\mathrm{RV} / \mathrm{LV}+\mathrm{S})$ demonstrated RV hypertrophy was not completely resolved in any group (Fig. 1g), the comparisons between $\mathrm{Hb}+\mathrm{HX}$ and $\mathrm{Hb}+\mathrm{HX}+\mathrm{GdCl}_{3}$ did not reach statistical significance. However, by comparing $\mathrm{HX}+\mathrm{GdCl}_{3}$ treated animals compared to HX alone, we noted greater mean PAP, medial thickening, and Fulton index (Supplemental Data Fig. 1). When combining all the treated and non-treated $\mathrm{GdCl}_{3}$ animals into two groups, data suggest eliminating circulating macrophages with $\mathrm{GdCl}_{3}$ slows the resolution of PAP, and 
(a)

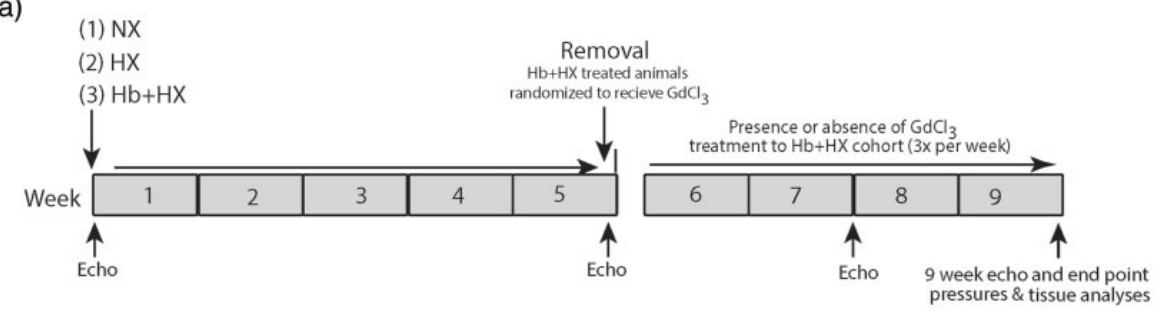

(b) Pulmonary artery

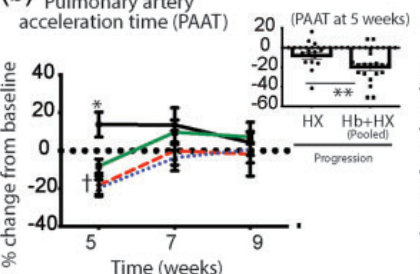

(c) Right ventricular wall thickness
diastole

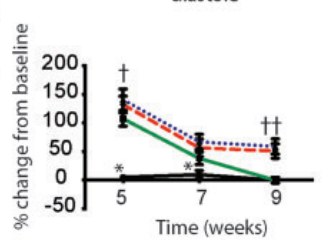

(d) Right ventricular wall thickness

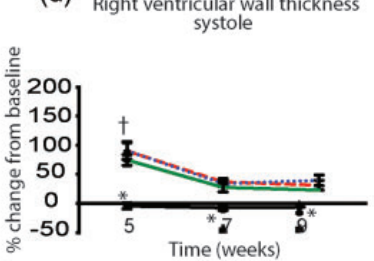

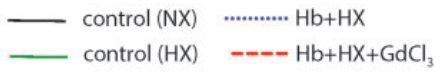

End point analysis at 9 weeks- 4 week of regression

(e) Mean Pulmonary Artery Pressure
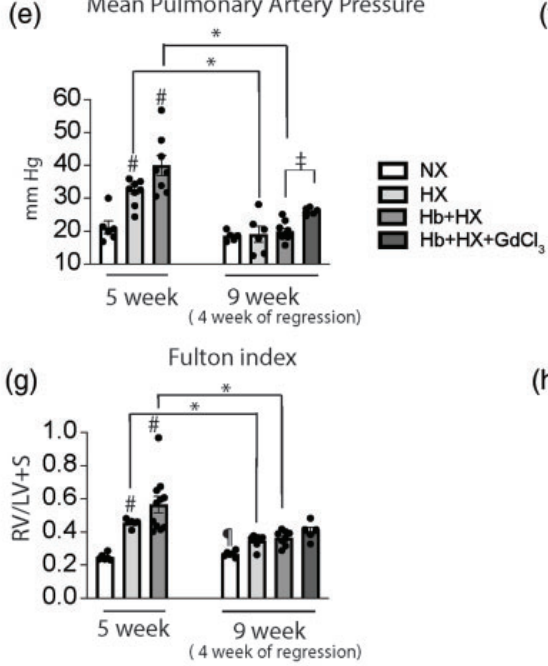

(f)

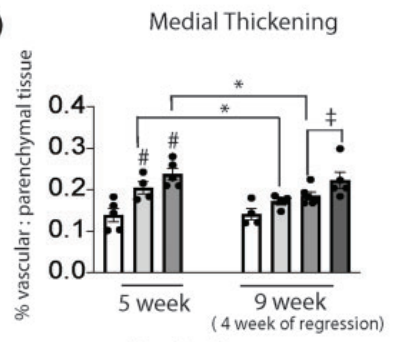

(h)

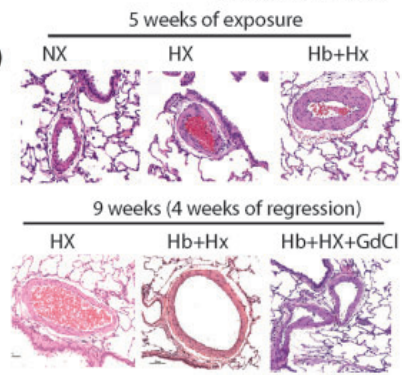

Fig. I. Cardiopulmonary analysis of the progression and the macrophage contribution in the resolution of hemoglobin ( $\mathrm{Hb}) \mathrm{plus}$ hypoxia (HX) mediated pulmonary hypertension. (a) Schematic of study design. Five weeks of exposure to $\mathrm{Hb}$ plus $\mathrm{Hx}$ followed by a four-week observational period after removal of $\mathrm{Hb}$ plus $\mathrm{HX}$. (b-d) Echocardiography measurements of the pulmonary artery acceleration time and right ventricular wall thickness in diastole and systole at five weeks after progressive $\mathrm{PH}$, and at two and four weeks after removal $\mathrm{PH}$ stimulus. (e-g) End point measurements of mean pulmonary artery pressure, pulmonary artery medial thickening, and right ventricular hypertrophy, and in a subset of rats sacrificed at five weeks and rats sacrificed after four weeks of recovery. (h) Microscopy visualization of H\&E stained lung tissue in rats after five weeks of progressive $\mathrm{PH}(\mathrm{HX}$ and $\mathrm{Hb}$ plus $\mathrm{HX}$ exposure) and after four weeks of recover. Normoxic rats were sacrificed at five weeks $(\mathrm{n}=3$ ) and nine weeks $(\mathrm{n}=3) .{ }^{\dagger} \mathrm{p}<0.05$ - five-week time point vs. seven- and nine-week time point; ${ }^{\dagger} \mathrm{p}<0.05 \mathrm{Hb}+\mathrm{HX}$ and $\mathrm{Hb}+\mathrm{HX}+\mathrm{GdCl} \mathrm{Hs}_{3}$. $\mathrm{HX}$ and NX cohorts at nine-week time point; ${ }^{\#} \mathrm{p}<0.05$ vs. NX at five weeks; ${ }^{*} \mathrm{p}<0.05$ - five-week vs. nine-week time points; $\neq \mathrm{p}<0.05 \mathrm{Hb}+\mathrm{HX}$ vs. $\mathrm{Hb}+\mathrm{HX}+\mathrm{GdCl}_{3}$ at nine-week time point; II $\mathrm{p}<0.05 \mathrm{NX}$ vs. all other groups at nine-week time point.

medial thickening, RV hypertrophy, at four weeks (Supplemental Fig. 1). While other factors are at play in the reversal of $\mathrm{PH}$, taken together these data suggest the circulating macrophage may work in concert with other cell types to either hasten the reversal process or are needed for complete resolution.
Gadolinium decreases cd 163 positive but increases cd68 positive macrophages in the lung after four weeks of reoxygenation

We demonstrate iron accumulation in the lung adventitial macrophages by five weeks of chronic $\mathrm{Hb}$ exposure with 
concomitant HX, and depletion of circulating macrophages prevented $\mathrm{PH}$ development in this model..$^{5-7}$ Initially, we sought to determine whether macrophages containing iron persist at four weeks after cessation of the stimulus. Tissue Perls-DAB staining of the lung revealed that some iron accumulation persists in the adventitia and parenchymal cells in the $\mathrm{Hb}+\mathrm{HX}$ rats (Fig. 2a). However, we cannot conclude if the early iron-containing macrophages survive in the lung for extended periods. As expected, we observed virtually no iron in the NX control animals and minimal iron in the $\mathrm{HX}$ and $\mathrm{Hb}+\mathrm{HX}+\mathrm{GdCl}_{3}$ cohorts (Fig. 2a). Macrophage activation and polarization to M2 phenotype promote $\mathrm{PH}$ and vascular remodeling. We characterized the macrophages in the lungs by staining for cd68, a pan macrophage marker, and cd163, a marker for the M2 phenotype. Levels of cd68, a pan macrophage marker, were lower in $\mathrm{HX}$ and $\mathrm{Hb}+\mathrm{HX}$ recovery groups but was significantly higher in $\mathrm{Hb}+\mathrm{HX}+\mathrm{GdCl}_{3}$ treated recovery group (Fig. 2b and c). Staining for cd163, a marker for M2 macrophage shows a significant decrease in the $\mathrm{Hb}+\mathrm{HX}$ and $\mathrm{Hb}+\mathrm{HX}+\mathrm{GdCl}_{3}$ recovery group but was not affected in the HX group compared to the HX group five-week time point (Fig. 2d-e). These data demonstrate that during regression, there is a decrease in the M2 macrophage phenotype. Interestingly there is also an increase in a subset of macrophages that appear resistant to $\mathrm{GdCl}_{3}$ treatment in the $\mathrm{Hb}+\mathrm{HX}$ group (Fig. 2b-c).

\section{Decreased IL-6 and ETI expression in rat lungs during recovery}

In rats exposed to $\mathrm{Hb}+\mathrm{HX}$ for five weeks, there is increased iron accumulation in adventitial macrophages that express mediators of hypoxia-driven PH, IL-6, and ET-1. ${ }^{7}$ Here, we examined if IL- 6 and ET-1 expression persisted in the residual iron overloaded macrophages after reoxygenation. Western blot analysis of lung lysates revealed decreased IL-6 in all groups of the recovery cohort. Furthermore, we observed IL-6 positive stain was predominantly in the media of distal pulmonary arteries of $\mathrm{Hb}+\mathrm{HX}$ treated rats during regression and not in the ironladen macrophages (Fig. 3a-c).

In contrast to IL-6, we observed robust ET- $1^{+}$cells in $\mathrm{Hb}+\mathrm{HX}$ rats located in both the adventitial and vascular compartments in distal pulmonary arteries (Fig. 3d-f). These areas were generally in the same locations as we observed and Iron $^{+}$cells, suggesting ET-1 persistence may be associated with iron-loaded macrophages (Fig. 3f). ET-1 was decreased in the $\mathrm{Hb}+\mathrm{HX}+\mathrm{GdCl}_{3}$ group, suggesting macrophages may be the dominant source of ET-1 in the lung (Fig. 3d). As expected, we observed minimal expression of $\mathrm{Il}-6^{+}$and $\mathrm{ET}-1^{+}$cells in both NX control rats and rats one month after chronic HX exposure. These results suggest that circulating macrophages increase ET-1 levels, but IL-6 levels were driven by hypoxia. Overall, these data demonstrate that the circulating macrophage influences $\mathrm{PH}$ mediators differentially during regression.

\section{Increased $\mathrm{HO}-\mathrm{I}$ expression during recovery}

Expression of HO-1 is considered protective in $\mathrm{PH}$ and other vascular diseases. We observed the persistence of HO-1 in iron positive cells in the vessel wall, in the $\mathrm{Hb}+\mathrm{HX}$ group like the macrophage expressing the $\mathrm{Hb}$ clearance phenotype in our previous studies. ${ }^{5-7}$ Interestingly, eliminating circulating macrophages during regression resulted in increased expression of $\mathrm{HO}-1^{+}$in cells throughout the lung (Fig. $4 \mathrm{a}-\mathrm{c}$ ). $\mathrm{HO}_{-} 1^{+}$cells are distributed throughout the vessel wall and highly expressed in the media of distal pulmonary arteries of the $\mathrm{Hb}+\mathrm{HX}+\mathrm{GdCl}_{3}$ rats (Fig. 4a-c), demonstrating a shift in lung HO-1 cellular expression with depletion of circulating macrophages. Congruent with immunofluorescence staining, Western blot analysis of whole lung tissue confirmed that HO-1 expression was significantly greater in the $\mathrm{Hb}+\mathrm{HX}+\mathrm{GdCl}_{3}$ treated rats (Fig. 4c). These results differ from those observed in the preventive study with $\mathrm{GdCl} 3$ treatment, where $\mathrm{HO}-1$ expression did not increase in the vessel wall. In the NX and HX cohorts, we noted HO1 expression was low in parenchymal and adventitial cells of the lung (Fig. 4a-c). Within the five-week main study, $\mathrm{Hb}+\mathrm{HX}$ caused diffuse fibrosis in peripheral pulmonary vessels consistent with muscularized remodeled lung vasculature (Fig. 4d). Fibrosis was not nearly as extensive in NX or the HX groups. During the four-week regression phase, $\mathrm{Hb}+\mathrm{HX}+\mathrm{GdCl}_{3}$ treated rats demonstrated diffuse interstitial and muscularized tissue fibrosis (Fig. 4d), suggesting that depletion of peripheral macrophages was not a factor in the progression of fibrosis during the four-week regression phase.

\section{Discussion}

Studies have shown that vascular remodeling reverses spontaneously upon reoxygenation in hypoxic $\mathrm{PH} .{ }^{13,14}$ Our $\mathrm{Hb}+\mathrm{HX}$ treatment model of $\mathrm{PH}$ shows a gradual reversal of $\mathrm{PH}$ in rats after withdrawal of $\mathrm{Hb}+\mathrm{HX}$ as measured at four weeks by a decrease in mean pulmonary artery pressures, RV hypertrophy, and medial thickening of arteries. Although additive in the initial five weeks of exposure, our data demonstrate cessation of $\mathrm{Hb}$ plus $\mathrm{HX}$ resolves as quickly as $\mathrm{PH}$ driven by $\mathrm{HX}$ alone $\mathrm{s}^{5-7}$ and also does not follow a fatal and unresolvable $\mathrm{PH}$ trajectory such as that seen in Sugen plus hypoxia PH rat model. ${ }^{15-17}$

Macrophages are a heterogeneous population, and two major phenotypes (M1 and M2) with opposing inflammatory and anti-inflammatory functions have been described in the lung. ${ }^{18}$ Studies suggest that macrophages isolated from PAH patients exhibit the M2 phenotype and are responsible for promoting smooth muscle proliferation and migration. ${ }^{19}$ An increase in M2/M1 ratio is thought 


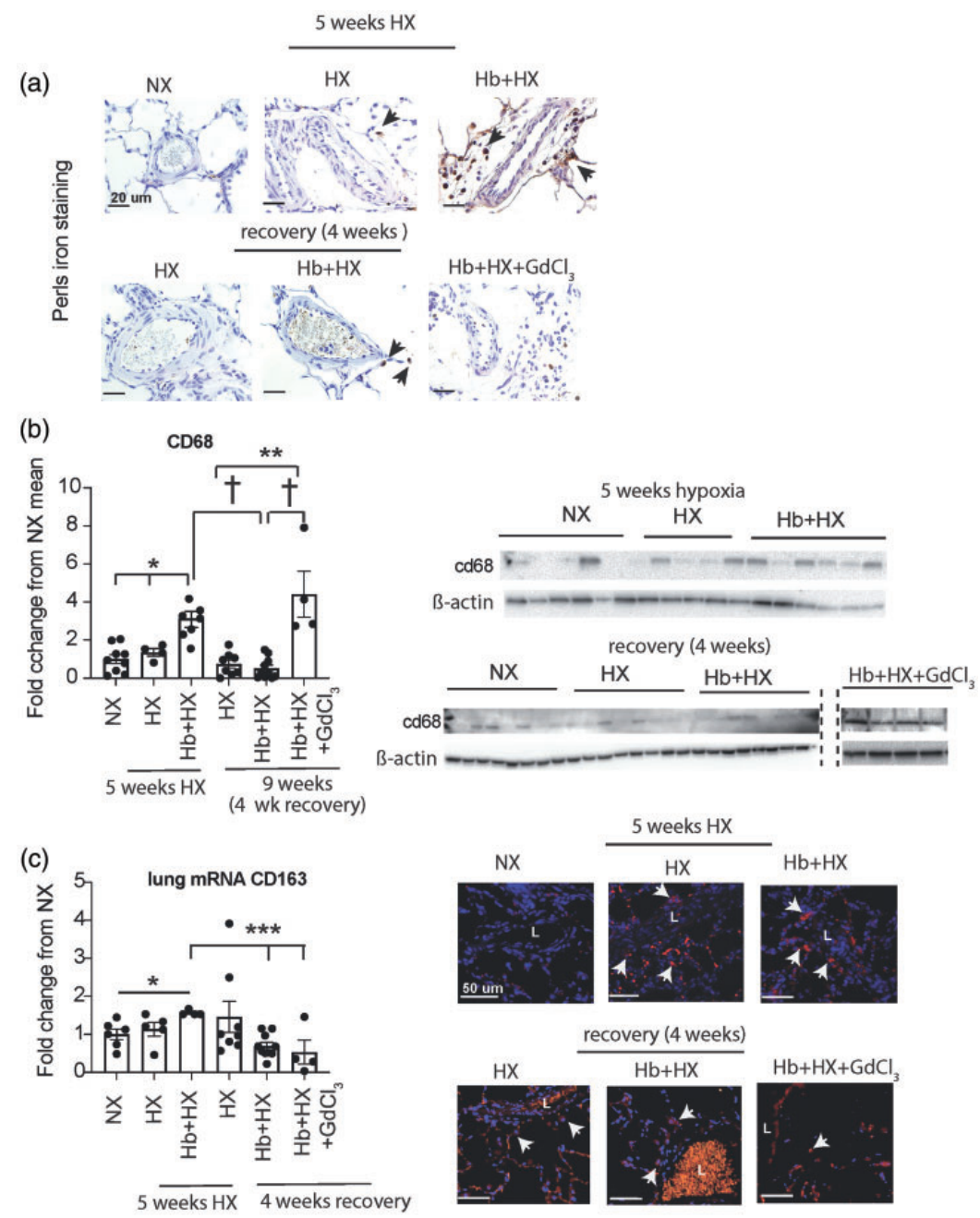

Fig. 2. Lung iron accumulation and presence of $\mathrm{cd} 68$ and $\mathrm{cdl} 63$ cell populations after five weeks of progressive $\mathrm{PH}$ and following four weeks of recovery. (a) Perls lung iron accumulation original magnification $60 \times$ Brown- iron, Black arrows- iron loaded cells (macrophages); (b) Western blot analysis of whole lung cd68; (c) mRNA quantification of cd I63 in whole lung tissue; (d) Immunohistochemistry microscopy visualization for cells expressing cdI63 in lung tissue sections of rats. Original magnification $40 \times$. Red- cells expressing cdl63. White arrows- positive cells.

to promote vascular remodeling. ${ }^{19}$ In our study cd163, mRNA levels were lower in both the $\mathrm{Hb}+\mathrm{HX}$ groups in the presence and absence of $\mathrm{GdCl}_{3}$. In mice with cd68 genetic deletion study, a decrease in macrophages in male mice altered the $\mathrm{M} 1 / \mathrm{M} 2$ ratio, and increased basal RV pressures were observed. ${ }^{19}$ In our study, cessation of $\mathrm{Hb}+\mathrm{HX}$ reduced the number of cd163 positive cells. However, we found a significant increase in cd68 positive macrophages in the $\mathrm{GdCl}_{3}$ treated cohort, showing increased pulmonary arterial pressures (Fig. 1). Our data cannot assess whether mean PAP increases are due to a skew in $\mathrm{M} 2 / \mathrm{M} 1$ ratio. The cd68 positive macrophages may be from a resident population since $\mathrm{GdCl}_{3}$ decreases circulating monocytemacrophage populations. There is evidence that alveolar macrophages can divide and self-renew without being replaced by stable circulating monocytes. ${ }^{20}$ Our group also has reported that the activation and expansion of alveolar and interstitial macrophages occur from different compartments during vascular remodeling at different time points in hypoxic $\mathrm{PH} .{ }^{21}$ Whether macrophages from different compartments contribute to the resolution phase requires a detailed characterization of the subsets of macrophages at different time points during the regression phase.

We observed that the deletion of circulating macrophages with $\mathrm{GdCl}_{3}$ in the recovery phase prevented the complete reversal of pulmonary arterial pressures and vascular remodeling, indicating that macrophages facilitate $\mathrm{PH}$ resolution (Fig. 1a-h; Supplemental Fig. 1). Macrophages, by increasing inflammation, contribute to $\mathrm{PH}$ progression. ${ }^{22}$ Numerous studies have shown that deleting macrophages in different preclinical models by chemical or genetic means prevents vascular remodeling and $\mathrm{PH}$ development. ${ }^{21,23}$ In our reversal study, the pro-inflammatory cytokine IL-6 returned to baseline levels upon withdrawal of $\mathrm{Hb}+\mathrm{HX}$, and the deletion of macrophages had no additional effect. However, ET-1 levels were high in the $\mathrm{Hb} / \mathrm{HX}$ group even 


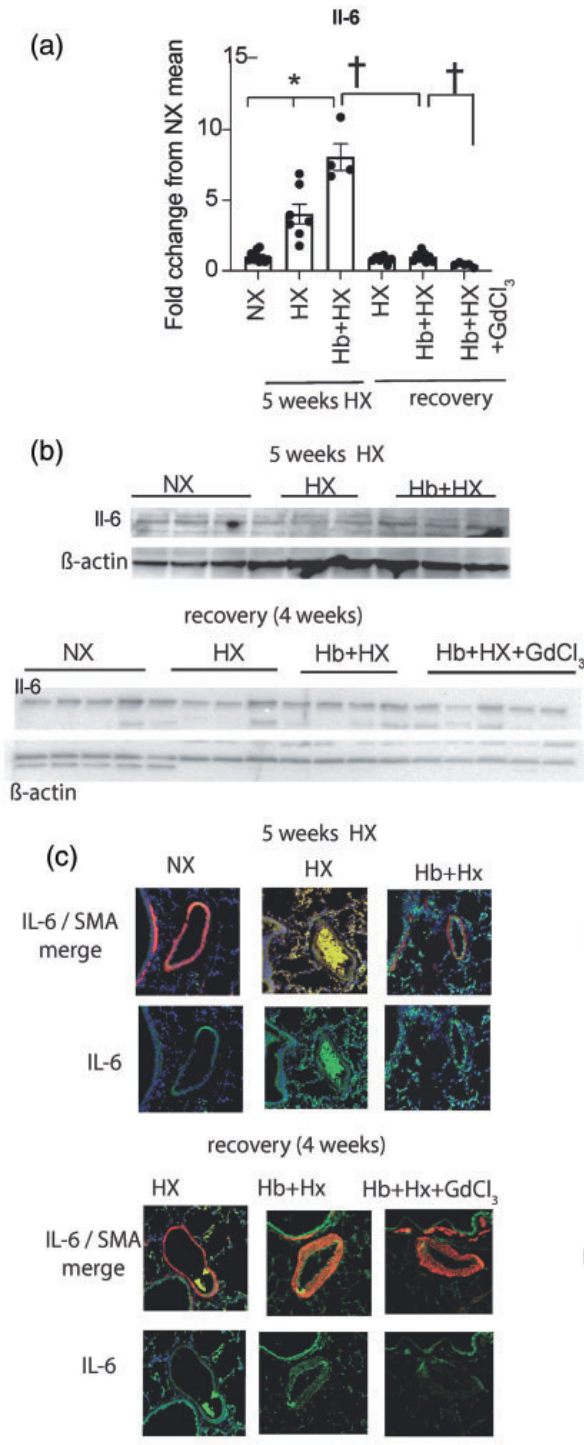

(d)
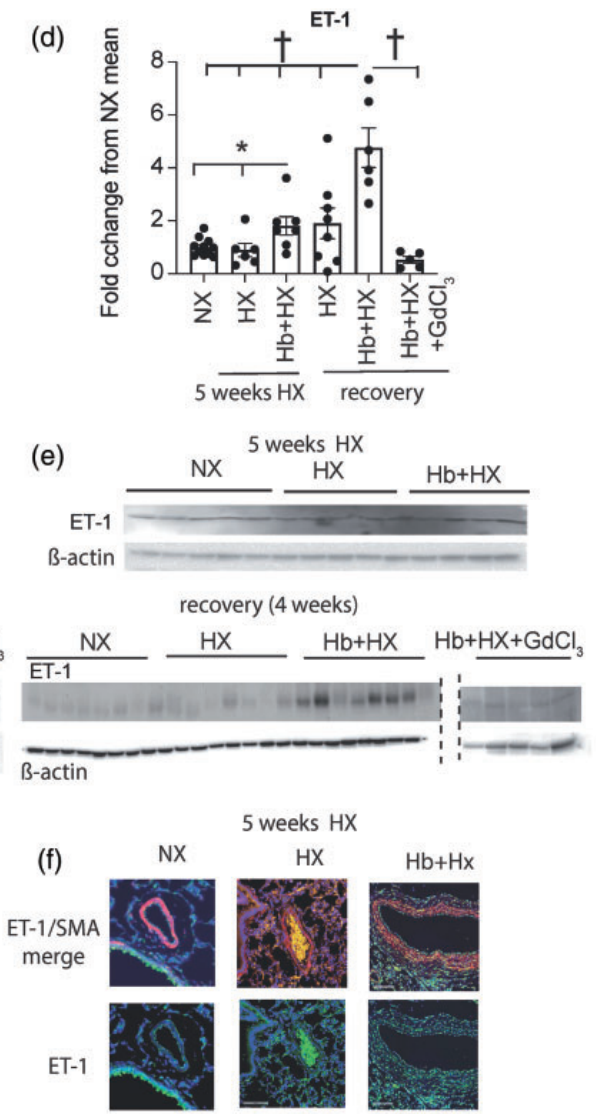

recovery (4 weeks)

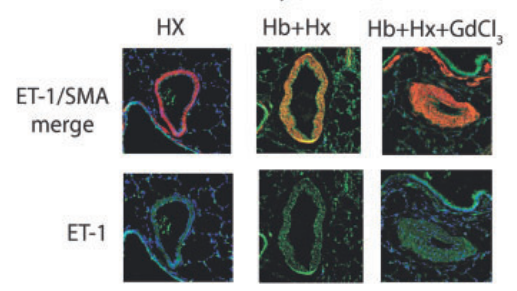

Fig. 3. Quantification and location of lung IL-6 and ET-I: (a) Western blot quantification of IL-6; (b) representative western blot of IL-6; (c) Immunohistochemistry visualization of location of lung and IL-6. Original magnification 40×. (d) Western blot quantification of ET-I; (e) representative western blot of ET-I; (f) Immunohistochemistry visualization of location of lung and ET-I. Original magnification $20 \times$ Immunohistochemistry visualization of location of lung $\mathrm{HO}-\mathrm{I}$ and ET-I. Original magnification $40 \times{ }^{\dagger} \mathrm{P}<0.05 \mathrm{vs}$. $\mathrm{Hb}+\mathrm{HX}$ at four weeks of regression; ${ }^{*} \mathrm{p}<0.05$ vs. $\mathrm{HB}+\mathrm{HX}$ at five weeks of progressive $\mathrm{PH}$; $* \mathrm{NX}$ vs. $\mathrm{Hb}+\mathrm{HX}$ by Students $\mathrm{t}$ test.

after withdrawal of $\mathrm{Hb} / \mathrm{HX}$, which was eliminated by $\mathrm{GdCl}_{3}$ treatment, suggesting that ET-1 was secreted by circulating macrophages. More recently, a study showed that macrophage-derived PDGF is a driver of PASMC growth and vascular remodeling. ${ }^{24}$ Therefore, in addition to proinflammatory markers macrophages may be the source of peptides that increase vascular constriction and remodeling.

Paradoxically, even with reduced IL-6 and ET-1, in the $\mathrm{GdCl}_{3}$ treated group, mean PAP and vascular remodeling were higher than NX mice. Other studies also show that macrophage depletion attenuates vascular remodeling and does not affect hemodynamics. ${ }^{25}$ The inhibition of Cx3cr1 in monocrotaline and hypoxia models of $\mathrm{PH}$ attenuated macrophage infiltration to the lung, preventing vascular remodeling but not improving hemodynamics. ${ }^{25,26}$ In cd68 KO mice, elevated pressures are observed in a normoxic environment suggesting a subset of macrophages are required to maintain homeostasis in the vascular system. ${ }^{19}$ Although in the $\mathrm{GdCl}_{3}$ treated group, we had increased levels of cd68 positive cells, whether they contribute to pressure cannot be concluded from our data. However, increased HO-1 expression in smooth muscle cells increases blood pressure by modulation of the NO pathway. ${ }^{27,28} \mathrm{We}$ observed a significant increase in HO-1 in the vessel wall of rats treated with $\mathrm{GdCl}_{3}$ (Fig. 4). Whether this contributes to the residual pressures will need further studies. This contrasts with other studies using transgenic mice where HO-1 is protective in $\mathrm{PH}^{29} \mathrm{HO}-1$ levels have shown to be 


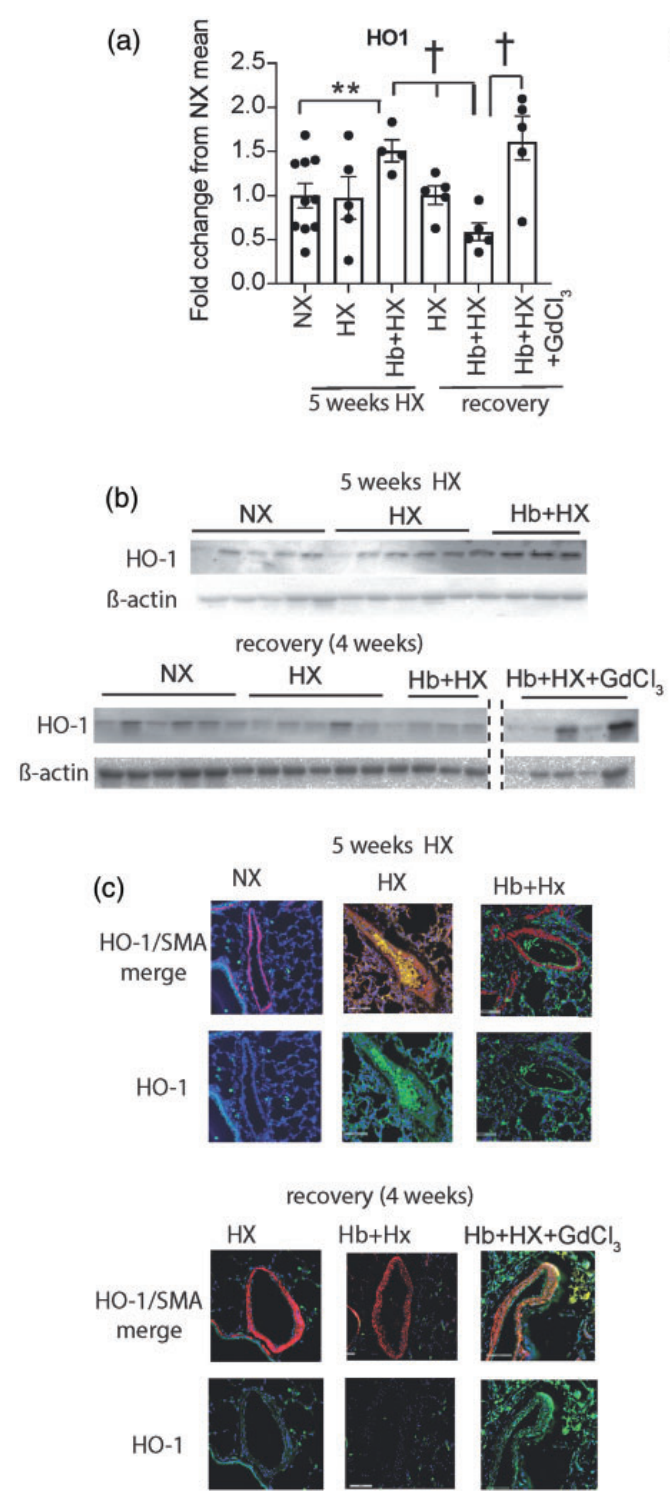

(d) Mason Trichrome Staining
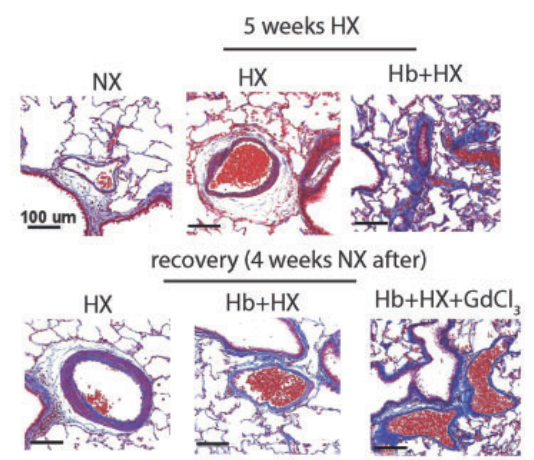

(1)

Fig. 4. Quantification and location of lung heme oxygenase-I (HO-I) and Masons Trichrome staining. (a) Western blot quantification of HO-I; (b) Representative western blot of $\mathrm{HO}-\mathrm{I}$; (c) Immunohistochemistry visualization of location of lung and $\mathrm{HO}-\mathrm{I}$. Original magnification $40 \times$; (d) Masons trichrome staining; Blue- collagen. Original magnification $20 \times .{ }^{\dagger} \mathrm{p}<0.05 \mathrm{vs}$. $\mathrm{Hb}+\mathrm{HX}$ at four weeks of regression; ${ }^{* *} \mathrm{NX}$ vs. $\mathrm{Hb}+\mathrm{HX}$ by Students $t$ test.

necessary for the beneficial effects of simvastatin in regression of established $\mathrm{PH}^{30}$

The heterogeneous population of myeloid cells in the lungs is essential for maintaining homeostasis and regulating inflammation, injury, and remodeling. ${ }^{31}$ Therefore, microenvironments may influence macrophage phenotype, thereby explaining many opposing macrophage functions. Evaluation of our data suggests that a subset of pro-PH macrophages is likely being recruited out of the lung and replaced by recruitment of other circulating macrophage phenotypes programmed to reverse the remodeling process. The current study's limitation is that we did not characterize a unique subset of macrophages expressing an apparent pro-resolution phenotype that reverses medial hypertrophy.
We have not yet used advanced techniques like flow cytometry to identify the populations of macrophages involved in the resolution. Advanced lineage tracing and single-cell transcriptomics will help determine the different macrophage populations in the progression and regression of PH. Follow-on studies will need to include metabolomics/ proteomic analysis on circulating and lung macrophages to characterize further the pro-resolution macrophage phenotype, an area we are actively working on. This knowledge could help develop novel therapies targeting monocyte/macrophages to trigger programming to counteract $\mathrm{PH}$ rather than aid the disease process.

The persistence of pro-PH macrophages after cessation of $\mathrm{Hb}$ and $\mathrm{HX}$ has implications in $\mathrm{PH}$ associated with 
hemolytic diseases such as human SCD. During 'crisis,' in patients with $\mathrm{SCD}$, the pulmonary vasculature is exposed to increased $\mathrm{Hb}$ levels and its degradation products (heme and iron) and tissue hypoxia. We propose that repeated cycles of these events cause accumulation of the pro-PH macrophages and unresolved inflammation, a key component of $\mathrm{PH}$ in the SCD population.

The data in the current study confirm previous observations that there is general plasticity of the pulmonary vasculature. ${ }^{15,32,33}$ In animal models, hypoxia-induced vascular remodeling can be reversed. ${ }^{16,17,34}$ Whether vascular remodeling can be reversed in human $\mathrm{PH}$ is not known. There are cases that severe PH has been resolved once the root cause was identified, isolated, and treated. ${ }^{35}$ Nevertheless, investigating how $\mathrm{PH}$ resolves in animal models may provide important insights into this critically important question.

\section{Conclusion}

In summary, supporting our original hypothesis we show that the circulating monocyte/macrophage population contributes to the resolution of unstable pulmonary hemodynamics and protein expression in rats after four weeks of recovery from active $\mathrm{Hb}$ plus hypoxia-mediated $\mathrm{PH}$ (five weeks of exposure to $\mathrm{Hb}$ plus hypoxia). While other factors are at play in the reversal of $\mathrm{PH}$, taken together these data suggest the circulating macrophage may work in concert with other cell types to either hasten the reversal process or needed for complete resolution. Further, our data show that despite resolution of medial hypertrophy, a subset of macrophages expressing a pro-PH phenotype characterized by high iron content and ET-1 expression persists in the pulmonary adventitia of distal pulmonary arteries. These data highlight the agility of the innate immune system responding during and after noxious stress on the pulmonary vasculature and may lead to therapies targeting the macrophage to aid in reversing $\mathrm{PH}$.

\section{Author contributions}

Designing research studies: VK, D Swindle, DIP, D Strassheim, MAF, ED, KRS, KH, RN, PWB, and DCI; Conducting experiments: VK, D Swindle, DIP, PWB, and DCI; Acquiring data: VK, D Swindle, DIP, PWB, and DCI; Analyzing data: VK, D Swindle, DIP, D Strassheim, MAF, ED, KRS, KH, RN, PWB, and DCI; Preparing figures: VK, DIP, PWB, and DIP; Writing manuscript: VK, D Swindle, DIP, D Strassheim, MAF, ED, KRS, KH, RN, $\mathrm{PWB}$, and DCI.

\section{Conflict of interest}

The author(s) declare that there is no conflict of interest.

\section{Funding}

The author(s) disclosed receipt of the following financial support for the research, authorship, and publication of this article: This work is supported by the U.S. Department of Health and Human
Services: 5P01HL014985-40, P0HL 152961, RO1HL 156526, R011HL 1598662, R01HL 161004.

\section{Guarantor}

DCI.

\section{ORCID iDs}

Kurt R Stenmark (D) https://orcid.org/0000-0001-6918-6411

Paul W. Buehler (D) https://orcid.org/0000-0003-0687-3008

David C. Irwin (D) https://orcid.org/0000-0002-1743-8266

\section{Supplemental Material}

Supplemental material for this article is available online.

\section{References}

1. Tuder RM, Stacher E, Robinson J, et al. Pathology of pulmonary hypertension. Clin Chest Med 2013; 34: 639-650.

2. Simonneau G, Montani D, Celermajer DS, et al. Haemodynamic definitions and updated clinical classification of pulmonary hypertension. Eur Respir J 2019; 53: 1801913.

3. Gordeuk VR, Castro OL and Machado RF. Pathophysiology and treatment of pulmonary hypertension in sickle cell disease. Blood 2016; 127: 820-828.

4. Rother RP, Bell L, Hillmen P, et al. The clinical sequelae of intravascular hemolysis and extracellular plasma hemoglobin: a novel mechanism of human disease. JAMA 2005; 293: 1653-1662.

5. Buehler PW, Baek JH, Lisk C, et al. Free hemolgobin induction of pulmonary vascular disease: evidence for and inflammatory mechanism. Am J Physiol Lung Cell Mol Physiol 2012; 303: 312-326.

6. Irwin DC, Hyen Baek J, Hassell K, et al. Hemoglobin-induced lung vascular oxidation, inflammation, and remodeling contribute to the progression of hypoxic pulmonary hypertension and is attenuated in rats with repeated-dose haptoglobin administration. Free Radic Biol Med 2015; 82: 50-62.

7. Redinus K, Baek JH, Yalamanoglu A, et al. An Hb-mediated circulating macrophage contributing to pulmonary vascular remodeling in sickle cell disease. JCI Insight 2019; 4: e127860.

8. Brissot P, Ropert M, Le Lan C, et al. Non-transferrin bound iron: a key role in iron overload and iron toxicity. Biochim Biophys Acta 2012; 1820: 403-410.

9. Highsmith FA, Driscoll CM, Chung BC, et al. An improved process for the production of sterile modified haemoglobin solutions. Biologicals 1997; 25: 257-268.

10. Lindsey ML, Kassiri Z, Virag JAI, et al. Guidelines for measuring cardiac physiology in mice. Am J Physiol Heart Circ Physiol 2018; 314: H733-H752.

11. Cavasin MA, Demos-Davies K, Horn TR, et al. Selective class I histone deacetylase inhibition suppresses hypoxia-induced cardiopulmonary remodeling through an antiproliferative mechanism. Circ Res 2012; 110: 739-748.

12. Kwon MS, Woo SK, Kurland DB, et al. Methemoglobin is an endogenous toll-like receptor 4 ligand-relevance to subarachnoid hemorrhage. Int J Mol Sci 2015; 16: 5028-5046.

13. Sakao S, Tatsumi K and Voelkel NF. Reversible or irreversible remodeling in pulmonary arterial hypertension. $A m J$ Respir Cell Mol Biol 2010; 43: 629-634. 
14. Sluiter I, van Heijst A, Haasdijk R, et al. Reversal of pulmonary vascular remodeling in pulmonary hypertensive rats. Exp Mol Pathol 2012; 93: 66-73.

15. Christou H, Hudalla H, Michael Z, et al. Impaired pulmonary arterial vasoconstriction and nitric oxide-mediated relaxation underlie severe pulmonary hypertension in the Sugen-hypoxia rat model. J Pharmacol Exp Ther 2018; 364: 258-274.

16. Stenmark KR, Meyrick B, Galie N, et al. Animal models of pulmonary arterial hypertension: the hope for etiological discovery and pharmacological cure. Am J Physiol Lung Cell Mol Physiol 2009; 297: L1013-L1032.

17. Sztuka K and Jasinska-Stroschein M. Animal models of pulmonary arterial hypertension: a systematic review and metaanalysis of data from 6126 animals. Pharmacol Res 2017; 125: 201-214.

18. Willis GR, Fernandez-Gonzalez A, Reis M, et al. Macrophage immunomodulation: the gatekeeper for mesenchymal stem cell derived-exosomes in pulmonary arterial hypertension? Int $J$ Mol Sci 2018; 19: 2534.

19. Zawia A, Arnold ND, West L, et al. Altered macrophage polarization induces experimental pulmonary hypertension and is observed in patients with pulmonary arterial hypertension. Arterioscler Thromb Vasc Biol 2021; 41: 430-445.

20. Hashimoto D, Chow A, Noizat C, et al. Tissue-resident macrophages self-maintain locally throughout adult life with minimal contribution from circulating monocytes. Immunity 2013; 38: 792-804.

21. Pugliese SC, Kumar S, Janssen WJ, et al. A time- and compartment-specific activation of lung macrophages in hypoxic pulmonary hypertension. J Immunol 2017; 198: 4802-4812.

22. Mindt M, Risse JM, Gruss H, et al. One-step process for production of $\mathrm{N}$-methylated amino acids from sugars and methylamine using recombinant Corynebacterium glutamicum as biocatalyst. Sci Rep 2018; 8: 12895.

23. Pugliese SC, Poth JM, Fini MA, et al. The role of inflammation in hypoxic pulmonary hypertension: from cellular mechanisms to clinical phenotypes. Am J Physiol Lung Cell Mol Physiol 2015; 308: L229-L252.

24. Ntokou A, Dave JM, Kauffman AC, et al. Macrophagederived PDGF-B induces muscularization in murine and human pulmonary hypertension. JCI Insight 2021; 6: e139067.
25. Florentin J, Coppin E, Vasamsetti SB, et al. Inflammatory macrophage expansion in pulmonary hypertension depends upon mobilization of blood-borne monocytes. $J$ Immunol 2018; 200: 3612-3625.

26. Amsellem V, Abid S, Poupel L, et al. Roles for the CX3CL1/ CX3CR1 and CCL2/CCR2 chemokine systems in hypoxic pulmonary hypertension. Am J Respir Cell Mol Biol 2017; 56: 597-608.

27. Imai T, Morita T, Shindo T, et al. Vascular smooth muscle cell-directed overexpression of heme oxygenase-1 elevates blood pressure through attenuation of nitric oxide-induced vasodilation in mice. Circ Res 2001; 89: 55-62.

28. Polizio AH, Santa-Cruz DM, Balestrasse KB, et al. Heme oxygenase-1 overexpression fails to attenuate hypertension when the nitric oxide synthase system is not fully operative. Pharmacology 2011; 87: 341-349.

29. Christou H, Morita T, Hsieh C-M, et al. Prevention of hypoxia-induced pulmonary hypertension by enhancement of endogenous heme oxygenase-1 in the rat. Circ Res 2000; 86: 1224-1229.

30. Lee TS, Chang CC, Zhu Y, et al. Simvastatin induces heme oxygenase-1: a novel mechanism of vessel protection. Circulation 2004; 110: 1296-1302.

31. Zaynagetdinov R, Sherrill TP, Kendall PL, et al. Identification of myeloid cell subsets in murine lungs using flow cytometry. Am J Respir Cell Mol Biol 2013; 49: 180-189.

32. Majesky MW, Horita H, Ostriker A, et al. Differentiated smooth muscle cells generate a subpopulation of resident vascular progenitor cells in the adventitia regulated by Klf4. Circ Res 2017; 120: 296-311.

33. McMurtry MS, Archer SL, Altieri DC, et al. Gene therapy targeting survivin selectively induces pulmonary vascular apoptosis and reverses pulmonary arterial hypertension. J Clin Invest 2005; 115: 1479-1491.

34. Dai Z, Zhu MM, Peng Y, et al. Therapeutic targeting of vascular remodeling and right heart failure in pulmonary arterial hypertension with a HIF-2alpha inhibitor. Am J Respir Crit Care Med 2018; 198: 1423-1434.

35. Balegadde AV, Vijan V and Thachathodiyl R. A case series of young patients with completely reversed severe pulmonary hypertension. J Clin Diagn Res 2017; 11: OR04-OR05. 